\title{
Polymer-based microfluidic device for measuring membrane protein activities
}

\author{
I. Hutter • E. Müller $\cdot$ P. M. Kristiansen • \\ S. Kresak $\cdot$ L. Tiefenauer
}

Received: 20 June 2012/ Accepted: 26 August 2012/Published online: 26 September 2012

(C) The Author(s) 2012. This article is published with open access at Springerlink.com

\begin{abstract}
Functional assays of membrane proteins are becoming increasingly important, both in research and drug discovery applications. The majority of current assays use the patch-clamp technology to measure the activity of ion channels which are over-expressed in cells. In future, in vitro assay systems will be available, which use reconstituted membrane proteins in free-standing lipid bilayers suspended in nano- or micrometer-sized pores. Such functional assays require (1) expression, purification and reconstitution of the membrane protein of interest, (2) a reliable method for lipid bilayer formation and membrane protein integration, and (3) a sensitive detection system. For practical applications, especially for automation, the reliable and controllable transport of fluids is essential. In order to achieve a stable free-standing lipid bilayer, a pore diameter in the micro- to nanometer range is essential. Novel microfluidic devices were developed by bonding a thick $(300 \mu \mathrm{m})$ polyether ether ketone foil, bearing a channel structure, to a thin $(12 \mu \mathrm{m})$ foil with a micropore of about $10 \mu \mathrm{m}$ diameter and then utilized for the
\end{abstract}

Electronic supplementary material The online version of this article (doi:10.1007/s10404-012-1061-0) contains supplementary material, which is available to authorized users.

I. Hutter · L. Tiefenauer $(\bowtie)$

Paul Scherrer Institute (PSI), Biomolecular Research (BMR), 5232 Villigen, Switzerland

e-mail: Louis.Tiefenauer@psi.ch

E. Müller · P. M. Kristiansen

Institute of Polymer Nanotechnology, University of Applied

Sciences and Arts FHNW, 5210 Windisch, Switzerland

S. Kresak

Max Planck Institute of Biophysics (MPIBP),

60438 Frankfurt a.M., Germany formation of stable, free-standing lipid bilayers within the pore. A bacterial voltage-gated potassium channel is integrated therein by fusion and the ion channel activity detected by voltage clamp.

Keywords Microfluidic - Fabrication · Polymer . Ion channel · Activity

\section{Introduction}

Proteins in the cell membrane are gatekeepers of various vital functions: they control the transport of ions and molecules across the cell membrane and they initiate-upon activation-the transduction of external signals to the intracellular biochemical machinery and thus regulate many cell functions. Due to their pivotal importance, membrane proteins are major targets for drugs which directly or indirectly regulate cell processes. Therefore, quantitative functional assays of membrane proteins are urgently needed in biological research as well as in drug discovery.

In order to assure full functionality of these fragile proteins, in vitro assays should be performed in a suitable environment, which mimics the biological membrane as closely as possible (Nielsen 2009). Free-standing lipid bilayers with a membrane protein of interest integrated therein allow us to directly monitor protein activity. For instance, the ion flow across membranes mediated by ion channels can be measured electrically. The development of such analytical devices comprises several crucial steps: (1) most importantly, making available the functional membrane protein of interest in a sufficient quantity, (2) establishing reliable procedures for stable lipid bilayer formation, (3) identifying robust methods to integrate the protein of interest into the lipid bilayer, and (4) developing 
sensitive detection methods. Furthermore, (5) the microfluidic system itself has three important functions: (a) reliable transport of fluids, (b) generation of stable lipid bilayer membranes with integrated membrane proteins separating two compartments and (c) integration of detection systems that allow electrophysiological measurements of membrane protein activities.

Silicon technology is highly reproducible and permits mass fabrication. Well-defined nanopores in ultrathin diaphragms have been fabricated and used for preparation of stable free-standing lipid bilayer membranes (Kresak et al. 2009; Danelon et al. 2006). They are stable for days in pores of diameters in the range of 200-400 nm (Han et al. 2007). The advantage of increased lipid bilayer stability in nanopores is opposed by the fact that the electrical capacitance of the ultrathin $(300 \mathrm{~nm})$ silicon nitride diaphragm is unacceptably high, hampering voltage-clamp measurements of single ion channels.

Alternatively, devices made of polymers for monitoring membrane protein activities have recently been presented by several groups (Suzuki and Takeuchi 2008; HiranoIwata et al. 2008; Estes et al. 2006; Malmstadt et al. 2006; Kawano et al. 2010). However, the combination of a microto nanometer-sized pore in a polymer foil of some micrometer thickness with a system of microfluidic channels in a macroscopic device remains a big challenge. For high throughput applications, the fabrication processes should be simple, cost-effective and enable mass production. In drug discovery, such devices are needed for the parallel detection of the ion channel activity of interest. In this paper, we describe a microfluidic device for the investigation of membrane proteins, which is entirely composed of polyether ether ketone (PEEK). PEEK, which is widely used for biomedical applications, provides a quite unique property spectrum: resistance to a wide range of chemicals and radiation, low moisture absorption, excellent barrier properties, electrical stability, a low dielectric constant and, most importantly, biocompatibility and low protein adsorption.

This PEEK-based microfluidic device can be used (1) for bilayer formation and (2) for the measurement of proteinmediated ion flow across lipid bilayer membranes. In bioanalytical devices, fragile lipid bilayers are generally formed just before the analysis, as their stability is limited. The development of microstructured materials and methods for handling small volumes has a long history (Zagnoni 2012).

The central part of a sensor device is the target molecule. In cells, ionic currents through over-expressed membrane channels or transporters of interest are routinely measured using patch-clamp techniques. The preparation of purified membrane proteins, however, includes many additional steps: expression, solubilization, purification and reconstitution (Demarche et al. 2011). The membrane protein can either be directly reconstituted within a planar lipid bilayer or in a liposome which is then fused by a second step into a preformed lipid bilayer. Upon application of an electrochemical potential across the planar proteobilayer, voltage-gated ion channels open within milliseconds. This opening can be detected as an ion current in the low pA-range. The archaebacterial voltage-gated $\mathrm{K}^{+}$channel (KvAP) is a robust model membrane protein used here to demonstrate the feasibility of the concept.

\section{Experimental}

\subsection{PEEK-foil device}

\subsubsection{Microstructuring and bonding}

APTIV $^{\circledR}$ PEEK $^{\mathrm{TM}}$ foils of $12 \mu \mathrm{m}$ (thin foil) and $300 \mu \mathrm{m}$ (thick foil) thickness, respectively, were kindly provided by Victrex ${ }^{\circledR}$ Polymer Solutions. Pores in the thin PEEK foil were ablated using a Duetto pico second pulsed laser from Time Bandwidth (15 W, pulses of $12 \mathrm{ps,} 355 \mathrm{~nm}$ ). Entry holes for the micro-channels were generated by ablating a circled line in the thin foil using $0.34 \mu \mathrm{J}$ at $1 \mathrm{MHz}$ and $300 \mathrm{~mm} / \mathrm{s}$. Full ablation of the PEEK-material by applying a pulse on demand for $60 \mathrm{~s}$ at $10 \mathrm{kHz}$ resulted in round micropores.

Micro-channels with rectangular cross sections (height $200 \mu \mathrm{m}$, width $500 \mu \mathrm{m}$ ) were prepared by hot embossing (HEX03, Jenoptik) of the thick foil using a polished brass stamp negative and applying a pressure of $200 \mathrm{kN}$ for $20 \mathrm{~min}$ at $155^{\circ} \mathrm{C}$. The channels showed good replication of the rectangular profile, which is an important prerequisite to achieve a good sealing and to ascertain laminar, homogeneous flow of liquids therein. Bonding of the thick to the thin foil was achieved by applying a pressure of $2 \mathrm{kN}$ at $140-150{ }^{\circ} \mathrm{C}$ for $16 \mathrm{~h}$. Plasma activation of the foils before bonding improved the bonding quality, but this was not investigated further.

\subsubsection{Device assembly}

The four entry/exit holes of the double Y-shaped microchannels address the micropore from the trans-compartment, whereas one additional port was put above the micropore addressing the cis-compartment (see Fig. 1). Four NanoPorts ${ }^{\mathrm{TM}}$ (Upchurch Scientific, USA) endowed with rubber fittings are set in the upper PMMA plate and connected to capillaries (1/16 inch, Upchurch Scientific). The transport of fluids was controlled by a NEMESYS ${ }^{\mathrm{TM}}$ pump system (Cetoni GmbH, Krobussen, Germany). The two $\mathrm{Ag} / \mathrm{AgCl}$ electrodes were positioned in one of the entry ports of the cis- and the trans-compartment, respectively. 


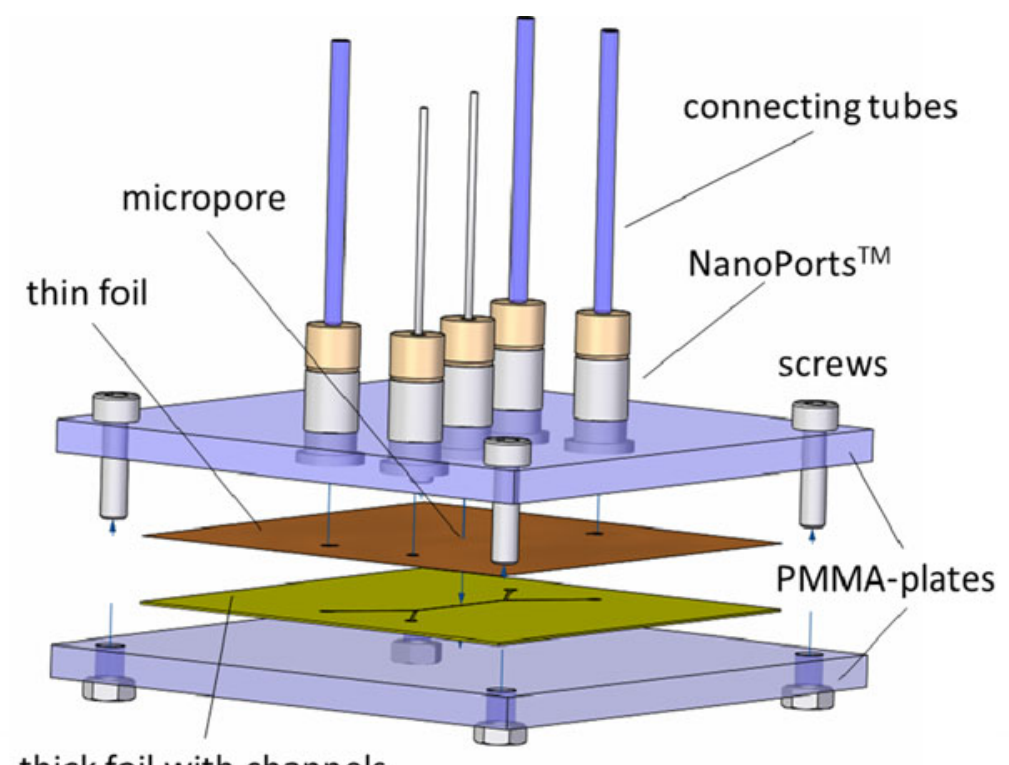

thick foil with channels
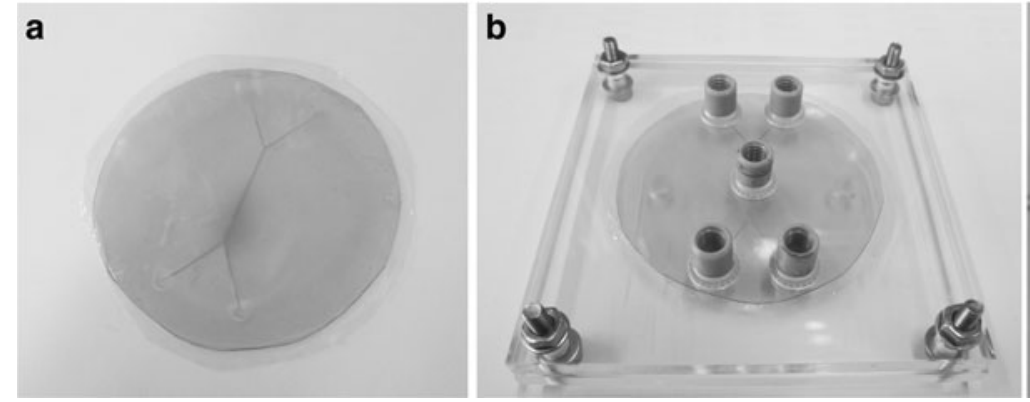

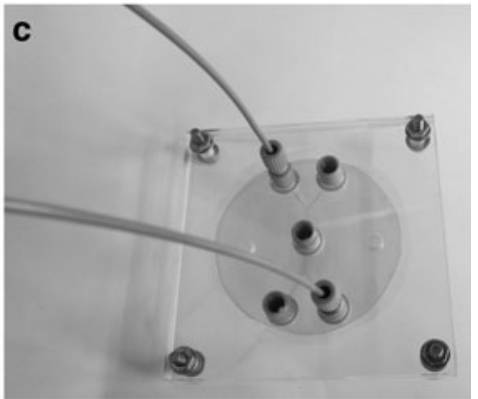

Fig. 1 Microfluidic device. The scheme (top) shows the setup of the microfluidic device. The $12 \mu \mathrm{m}$ thin PEEK foil containing the $10 \mu \mathrm{m}$ wide micropore is bonded to the $300 \mu \mathrm{m}$ thick PEEK foil containing the microfluidic channel structure (a) and the bonded sheets

\subsection{Protein expression, purification and reconstitution}

KvAP (from Aeropyrum pernix) cloned into pET-26b $(+$ ) (Ruta et al. 2003) was provided by the lab of Klaus Fendler at MPIBP Biophysics in Frankfurt. The KvAP channel protein was expressed in C43 DE3 cell cultures grown in LB-Lennox medium supplemented with $10 \mathrm{mM} \mathrm{BaCl}_{2}$ on induction with $1 \mathrm{mM}$ isopropyl- $\beta$-D-thiogalactopyranoside (IPTG) (at OD 0.6) for $5 \mathrm{~h}$ at $37^{\circ} \mathrm{C}$. Cells were har-

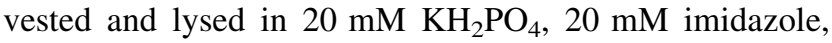
$500 \mathrm{mM} \mathrm{KCl}, \mathrm{pH} 8.0$ and $10 \%$ glycerol (v/v) containing protease inhibitors (protease inhibitor cocktail completely EDTA-free, Roche) and deoxyribonuclease I (crude preparation from bovine pancreases, Sigma-Aldrich). Protein was then extracted from the cell lysate at $4{ }^{\circ} \mathrm{C}$ overnight in the above solution by adding decylmaltoside (DM from Anatrace Inc., Maumee OH, USA) to a final concentration of $2 \%$, purified on a Ni-column (HisTrap ${ }^{\mathrm{TM}}$ ), and eluted with $20 \mathrm{mM} \mathrm{KH}_{2} \mathrm{PO}_{4} \mathrm{pH} 8.0,400 \mathrm{mM}$ imidazole, $500 \mathrm{mM}$ sandwiched between two PMMA plates (b). Pores (1.5 mm diameter) at the channel ends are connected via PEEK tubes (c) to an automated pump system

$\mathrm{KCl}, 10 \%$ glycerol (v/v), and $0.2 \% \mathrm{DM}$. To remove the imidazole a buffer exchange was performed by a PD10 column and the protein eluted with $20 \mathrm{mM} \mathrm{KH}_{2} \mathrm{PO}_{4}, \mathrm{pH}$ 8.0, $500 \mathrm{mM} \mathrm{KCl}, 10 \%$ glycerol (v/v), and $0.2 \% \mathrm{DM}$. The yield from $12 \mathrm{~L}$ cultures was about $0.6 \mathrm{mg}$ protein. The protein after IMAC-column was essentially pure (Fig. S5) as confirmed by sodium dodecyl sulfate (SDS) polyacrylamide gel electrophoresis (PAGE). The tetrameric protein was reconstituted with 1,2-diphytanoyl-sn-glycero-3phosphocholine (DPhPC, Avanti Polar Lipids, Alabaster, AL, USA) with a protein/lipid ratio of 1:20 (w/w) using $100 \mathrm{mg}$ Biobeads SM2 (Biorad) in $200 \mu \mathrm{L}$ solution resulting in proteoliposomes.

\subsection{Lipid bilayer formation and protein integration}

To form lipid bilayers across the micropore in the microchannel, buffers and lipids dissolved in decane were transported through the microfluidic device via tubes 
connected to a NEMESYS pump system, which was externally controlled by its software. The device was first filled with buffer (150 mM KCl, $10 \mathrm{mM}$ 4-(2-hydroxyethyl)-1piperazineethanesulfonic acid (HEPES), pH 7.4) by applying negative pressure with the pumps (at a speed of $0.2 \mu \mathrm{L} \mathrm{s}^{-1}$ ), $\mathrm{Ag} / \mathrm{AgCl}$ electrodes put in place and the current through the micropore measured. 2-3 $\mu \mathrm{L}$ of the lipid mixture 1,2-dioleoyl-glycero-3-phosphoglycerol and 1-palmitoyl-2oleoyl-glycero-3-phosphoethanolamine (DOPG:POPE, 1:3 $(\mathrm{v} / \mathrm{v}), 10 \mathrm{mg} \mathrm{mL}^{-1}$ in decane) were directly injected into the micro-channel through a valve and transported to the micropore by applying negative pressure. Moving lipids across the pore two to three times, usually resulted in the formation of a lipid bilayer membrane (the so called painting method). Formation of a lipid bilayer membrane was confirmed through impedance spectroscopy, voltage breakdown and peptide or protein integration (see below). To incorporate the KvAP ion channel protein, a lipid bilayer was painted and the bilayer positioned horizontally. $2 \mu \mathrm{L}$ of KvAP reconstituted in DPhPC liposomes were added from above, followed by $2 \mu \mathrm{L}$ of a $3 \mathrm{M} \mathrm{KCl}$ solution to force the proteoliposomes to fuse with the bilayer. If channels could not be detected after a few minutes, the bilayer was destroyed and a new one was formed.

\subsection{Electrical measurements}

Voltage breakdown experiments were performed by applying a linear voltammetry scan using an Autolab Potentiostat PG12 (Ecochemie, Utrecht, The Netherlands) equipped with a FRA module. The potential across the membrane was increased from 0 to $3,000 \mathrm{mV}$ at a scan rate of $10 \mathrm{mVs}^{-1}$ and a step potential of $1 \mathrm{mV}$. If membranes did not break, they were considered as lipid plugs. Single lipid bilayer formation was further confirmed through impedance spectroscopy. Samples were positioned in a Faraday cage and electrical impedance spectra (EIS) recorded from $1 \mathrm{MHz}$ to $0.01 \mathrm{~Hz}$ at $0 \mathrm{~V}$ offset potential, applying $10 \mathrm{mV}$ signal amplitude between the two $\mathrm{Ag} / \mathrm{AgCl}$ electrodes. In a further series of experiments, lipid bilayer membrane formation was confirmed by adding the peptide melittin (Fluka, Buchs, Switzerland) to an end concentration of $10 \mu \mathrm{g} \mathrm{mL}^{-1}$. A continuous potential of $80 \mathrm{mV}$ was applied across the membrane and the membrane permeability monitored.

KvAP activity was measured with a single channel amplifier (Axopatch 200B, Molecular Devices, Sunnyvale, CA, USA), applying a potential alternatingly in the range of +200 to $-200 \mathrm{mV}$. The $\mathrm{Ag} / \mathrm{AgCl}$ electrodes were made from $\mathrm{Ag}$ wires (diameter $1 \mathrm{~mm}$ ) and daily conditioned using $2.6 \%$ Javelle water $(\mathrm{NaClO})$.

\section{Results and discussion}

\subsection{Microfluidic device}

A PEEK-based microfluidic device (Fig. 1) was fabricated using three key fabrication technologies: (1) laser ablation in a thin $(12 \mu \mathrm{m})$ PEEK foil to generate micropores, (2) hot embossing of microstructures in a thick $(300 \mu \mathrm{m})$ PEEK foil and (3) thermal bonding of the two structured foils to form a closed channel structure. The bonded foils (Fig. 1a) were then assembled between two PMMA plates (Fig. 1b), connected via PEEK tubes to pumps and the fluid transport automatically controlled (Fig. 1c). Thus, only PEEK surfaces come into contact with the organic and aqueous solvents. This biocompatible material is resistant to most solvents and shows low moisture uptake and adsorption of biomolecules, an excellent prerequisite for the intended bioanalytical application.

\subsubsection{Micropore fabrication}

The size and the surface quality of the pores are important factors for stable free-standing lipid bilayers suspended therein. The pulsed laser method used here guaranties the generation of smooth, high quality surfaces on the backside (Fig. S1). A controlled ablation of the polymeric material is optimal, if only few pulses are picked (e.g. $P P=16$
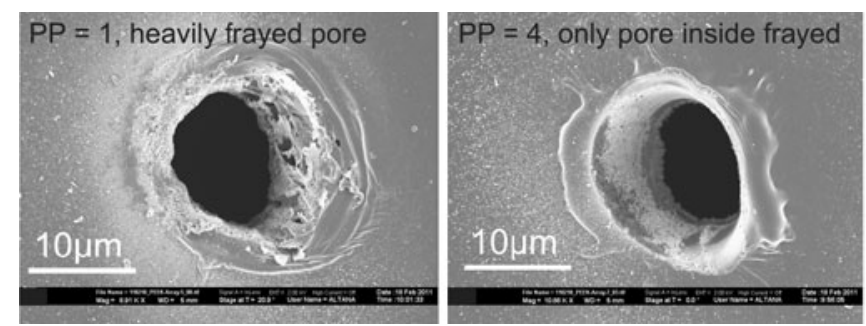

Fig. 2 Fabrication of micropores. Scanning electron microscopy (SEM) pictures of micropores (scale bars $10 \mu \mathrm{m}$ ) fabricated using ps-pulsed laser ablation. Micropores were generated in the $12 \mu \mathrm{m}$ thin PEEK foil by releasing different numbers of energy packages from a
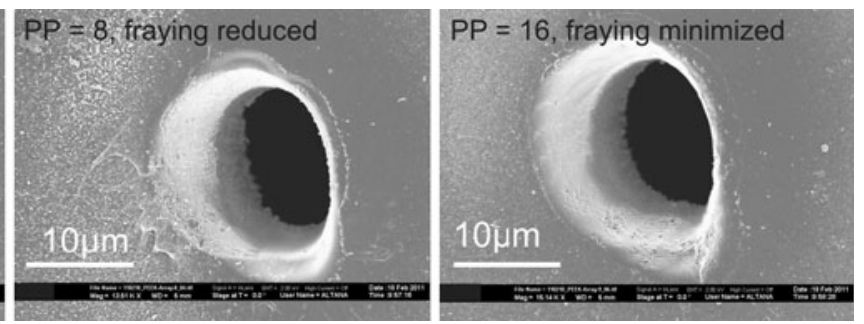

laser, applying the pulse picking (PP) method. In the first experiment $(\mathrm{PP}=1)$ all available pulses were shot to the foil, whereas in the last experiment only each sixteenth pulse was picked $(\mathrm{PP}=16)$ 
denotes that the sample is subjected to every 16th laser pulse only) as shown in Fig. 2. The pulse picking method allows control of the thermal energy input thus avoiding uncontrolled ablation and, as a consequence, leads to smooth rims and flat surfaces on the back side (Fig. 1S). The diameter of the pores can be as small as $5 \mu \mathrm{m}$, small enough to achieve sufficiently stable bilayers (see Fig. 7).

\subsubsection{Microfluidic channel preparation}

Hot embossing is a common, robust technique for high fidelity replication of micro- and nanostructures into polymers. For making microchannels in the thick PEEK foil, a stamp with the negative layout was pressed into the thicker foil. Since PEEK has a high glass transition

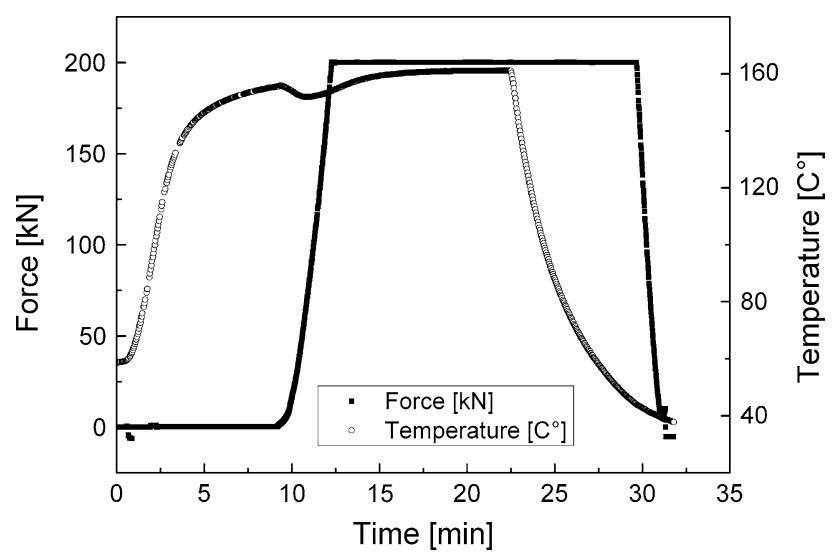

Fig. 3 Temperature and force profile of the hot embossing process. The channel structure was generated by hot embossing. After heating the PEEK foil to a temperature above $T_{\mathrm{g}}$, the channel structure was replicated by pressing a brass plunger into a $300 \mu \mathrm{m}$ thick PEEK foil for $10 \mathrm{~min}$ at $155^{\circ} \mathrm{C}$, applying a force of $200 \mathrm{kN}$. The embossed structure was then cooled down to $40{ }^{\circ} \mathrm{C}$ before releasing the pressure force to allow de-molding without deformation of the embossed structure. The brass plunger (negative structure) was mechanically fabricated through rotating and milling a brass plate temperature $\left(T_{\mathrm{g}}=145^{\circ} \mathrm{C}\right)$, the structure transfer was carried out at temperatures above $T_{\mathrm{g}}$. In a series of trials, the two major parameters, temperature and pressure, were varied and the quality of the resulting embossed channel structures checked by SEM imaging. We found that the procedure shown in Fig. 3 gave the best results: first increasing the temperature slightly above the transition temperature and when this point is reached, applying a high force. After holding the force for $10 \mathrm{~min}$ at fairly constant temperature, the sample was cooled down under pressure in order to avoid a deformation of the embossed channel structures upon demolding. The resulting channels show a rectangular shape with vertical side walls and sharp rims (Fig. 4), important features for a successful subsequent bonding. The embossing time needed is still quite long due to the depth of the channels $(200 \mu \mathrm{m})$ and can be optimized. For mass fabrication of microfluidic structures within PEEK, injection molding in a cast would be the method of choice. The entire volume of a double Y-shaped channel is in the low microlitre range for a channel width of $500 \mu \mathrm{m}$ and depth of $200 \mu \mathrm{m}$, which can be further reduced. However, if aqueous and organic fluids are subsequently transported, as is the case in the painting method used here, it has to be considered that the pressure drop $\Delta p$ substantially increases upon reduction of the hydrodynamic diameter $D_{\mathrm{H}}$ (scaling according to $\Delta p \sim D_{\mathrm{H}}^{-2}$ ).

\subsubsection{Bonding the micro-structured foils}

PEEK is an inert material with a glass transition temperature of $145^{\circ} \mathrm{C}$ and a melting point of $343{ }^{\circ} \mathrm{C}$. In order to achieve effective adhesive-free bonding, the polymer temperature needs to be raised just above $T_{\mathrm{g}}$ to allow sufficient interdiffusion of polymer chains between the two foils to be bonded. At the same time, recrystallization of the polymer may occur in this temperature regime, which may render the process slightly more difficult. Thus, a
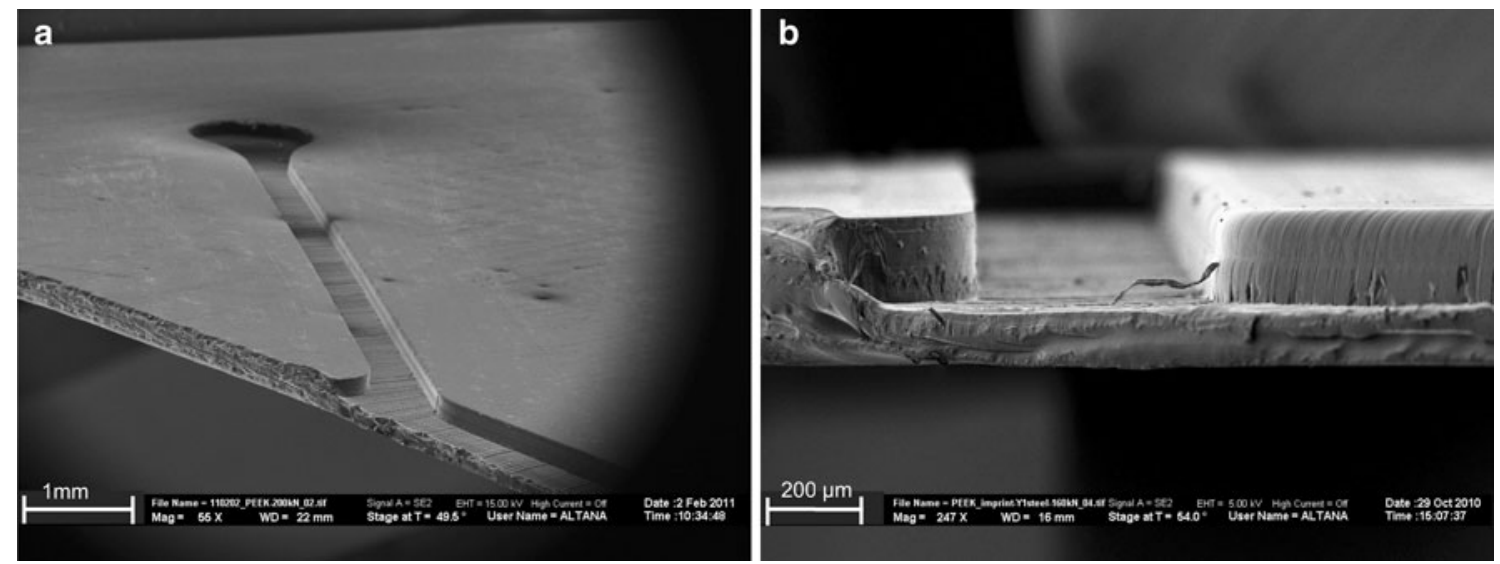

Fig. 4 SEM pictures of channel structures generated through hot embossing with a brass plunger 


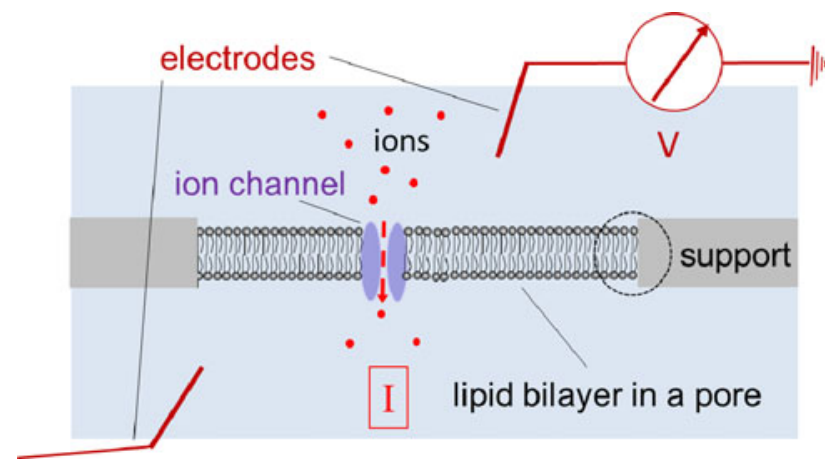

Fig. 5 Scheme of an ion channel measurement. A lipid bilayer suspended in a micropore separates the lower compartment, the microfluidic channel, from the upper compartment above the micropore (see Fig. 1 for electrode placements). The electrogenic activity of the integral membrane protein, connecting the two compartments, can then be determined by voltage-clamp measurements (see Fig. 8). I means protein-mediated ion current flow, the dotted circle the annulus zone

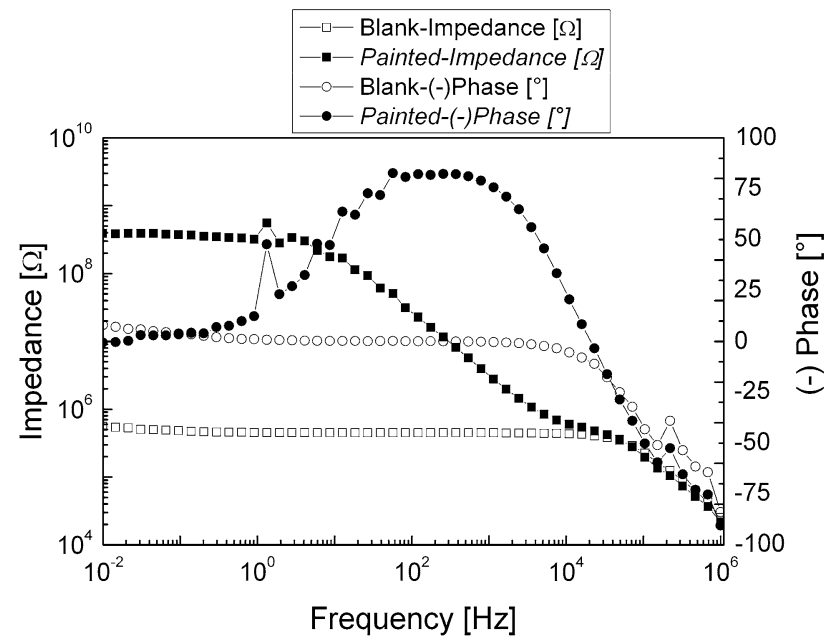

Fig. 6 Bilayer formation. Electrochemical impedance spectroscopy (EIS) is frequently used to confirm bilayer formation (e.g. Han et al. 2007). The spectra demonstrate a good sealing of the painted membrane, a prerequisite for single molecule detection. Blank measurement before painting

careful choice of temperature and pressure is required in order to achieve a defect-free and tight contact between the two foils, simultaneously preserving the cross-sectional shape of the microchannels that should not be impaired by the bonding process. In a series of experiments, the following suitable conditions were established (Fig. S2): applying $2 \mathrm{kN}$ pressure for $12 \mathrm{~h}$ at $145{ }^{\circ} \mathrm{C}$, followed by decreasing the temperature and simultaneously increasing the pressure to $3 \mathrm{kN}$ for $20 \mathrm{~min}$. This procedure resulted in sufficiently accurate devices (see Fig. 1a), having channels with a rectangular cross section (Fig. S3). Alignment of the micropore in the thin PEEK foil to the channels of $500 \mu \mathrm{m}$ width, was achieved manually before bonding (Fig. S4b).
Alignment of smaller channels will be more demanding and may require alignment markers. For practical applications, the above reported bonding time is far too long. However, using laser welding for bonding of the foils promises significant reduction of the bonding time. In addition, plasma activation of the PEEK surfaces prior to bonding will considerably enhance the adhesion between the two foils and, in addition, may likely reduce the bonding time. In summary, three critical parameters for the thermal bonding of PEEK to PEEK have been identified and determined for this prototype: temperature, pressure and time. Successful thermal bonding of the PEEK prototype setup could be achieved at temperatures just above $T_{\mathrm{g}}$ $\left(145-150{ }^{\circ} \mathrm{C}\right.$ ), applying low pressure forces (few $\mathrm{kN}$ ) and sufficiently long bonding times $(>10 \mathrm{~h})$.

\subsubsection{PEEK-microfluidic device assembly}

The bonded PEEK foils have been assembled to a functional microfluidic device using two PMMA-plates and the nanoport system using fitting rings (Fig. 1c). Connection to the syringe pump system using PEEK ports allows air-tight transport of fluids. Lipids were added to the system through injection of some microlitre of lipid solutions through a sixfold valve.

\subsection{Membrane formation and ion channel measurements}

In principle, the conductance of a membrane separating two compartments increases when voltage-gated ion channels are open after applying a potential (Fig. 5). Ion current events are monitored over time.

\subsubsection{Automated formation of lipid bilayers in micropores}

The commonly used painting method was applied to generate stable lipid bilayers. This process can be monitored using electrical impedance spectroscopy (EIS) (Fig. 6). Upon painting, an increase of more than two orders of magnitude in the impedance value at low frequencies and a shift of the phase to $90^{\circ}$ were observed, two indications for bilayer formation (Han et al. 2007). As further explained in that study, bilayer formation can be confirmed by an increase of the total capacitance. Artificial lipid bilayers, with a thickness of $4 \mathrm{~nm}$, have a specific capacitance of around $1 \mu \mathrm{Fcm}^{-2}$. The area of a lipid bilayer membrane in a pore of $10 \mu \mathrm{m}$ diameter is $8 \times 10^{-9} \mathrm{~cm}^{2}$ and the expected bilayer capacitance less than $1 \mathrm{pF}$, a value hardly detectable by EIS. Therefore, bilayer formation was further confirmed by adding melittin, a pore forming peptide. After the addition of melittin to the solution, the peptide adheres to the bilayer membrane and spontaneously inserts itself 

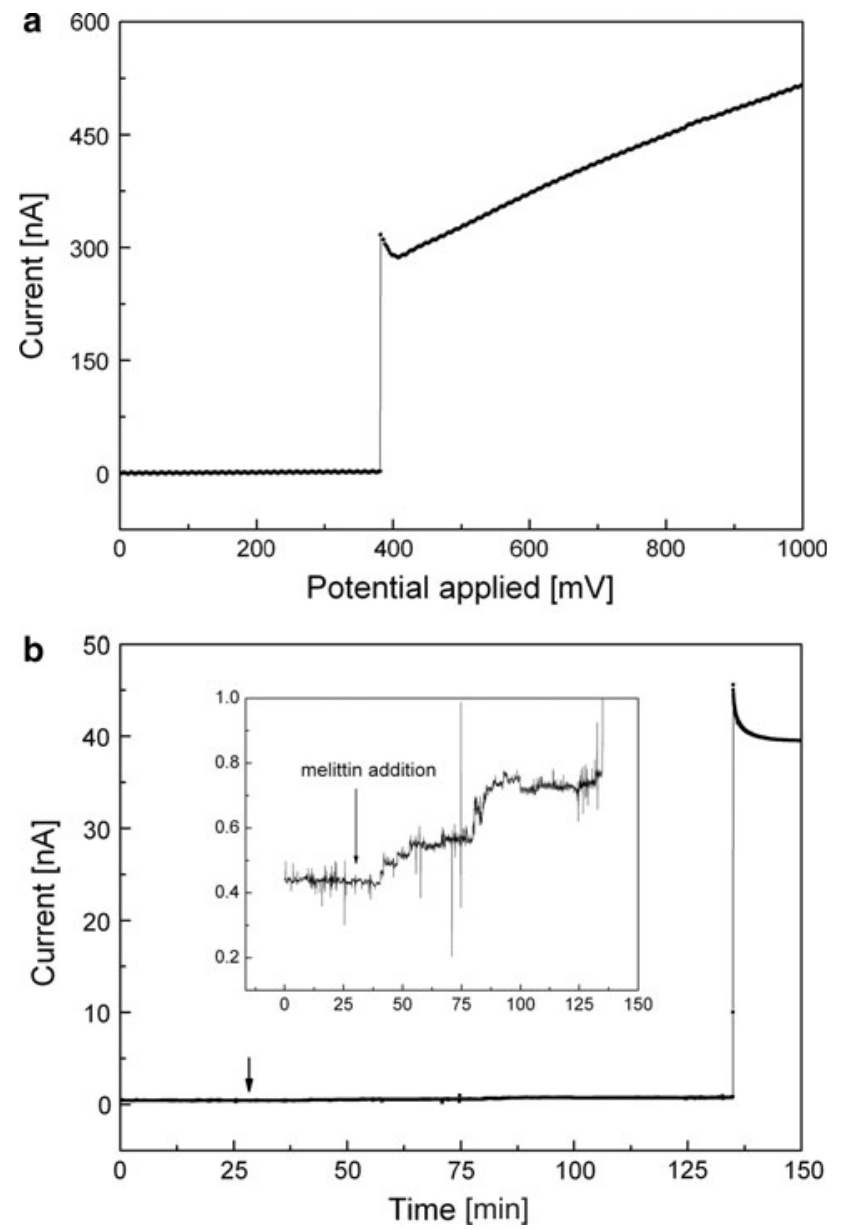

Fig. 7 Characterization of the lipid bilayer formed. The stability of a bilayer suspended in a micropore is assessed by steadily increasing the voltage and finding the point of a sudden increase of current where the bilayer breaks (a). Bilayer formation is further confirmed by monitoring electrochemically peptidic pores which appear after a lag time of about $10 \mathrm{~min}$ upon melittin addition (arrows). When too many pores are formed, the bilayer breaks (b). $x$ and $y$ labels are valid for the figure and the insert

into the lipid bilayer membrane. Four peptide molecules together form a pore in the membrane (Studer et al. 2009), which can be detected as intermediate current peaks after a lag time of about $10 \mathrm{~min}$ (Fig. $7 \mathrm{~b}$ ).

For the practical use of such devices, stability of the notoriously fragile membrane is a major issue. In this study, the stability of painted lipid bilayers was assessed according to a previously reported method (Meier et al. 2000). The breaking point of bilayers was assessed by continuously increasing the electrical potential to a point where it breaks and a rapid increase in current is observed. This potential provides a measure of bilayer stability. Breaking does not occur always at the same point; here the lowest observed breaking point (Fig. 7a) in a series of six experiments is shown, with the highest ones being above $1,000 \mathrm{mV}$. This value is reasonably high for practical applications and in full agreement with the published data of about $400 \mathrm{mV}$ for a Teflon support with a pore of similar size (Mayer et al. 2003).

The stability of the lipid bilayer may be partially related to moving lipids dissolved in a residual solvent reservoir in the annulus (see Fig. 5) of the pore rim. Furthermore, surface properties of the support, such as roughness, shape and contact angle can influence the stability and sealing of suspended bilayers. From experiments using several prototype devices, we conclude that pore diameters ranging from 5 to about $12 \mu \mathrm{m}$, even if not circular, are suitable for lipid bilayer formation. The smooth surface around the pores probably favors a good sealing but this has not yet been investigated in detail. However, in this study the mentioned factors have not been systematically investigated. It is assumed that the most important factor for stable lipid bilayers is a hydrophobic surface, i.e. a contact angle above $90^{\circ}$. The PEEK foil used here has a contact angle close to this value. Bilayers in nanometer-sized pores in silicon nitride membranes have been shown to be stable for days (Tiefenauer and Studer 2008). A sufficiently high break-down voltage value in the micrometer-sized polymer support could be demonstrated in this study using pores of about $10 \mu \mathrm{m}$ diameter. Whereas the electrical capacitance of thick foils is low and their mechanical stability is high, pores of smaller diameters can be made in thinner foils resulting in enhanced bilayer stability. Considering these contradictory factors, a pore diameter of $5-10 \mu \mathrm{m}$ is suitable for practical applications. In summary, a sufficient stability of the bilayers in micropores has been achieved, which can be further improved.

A final experiment to proof bilayer formation (Fig. 7b) was the monitoring of melittin incorporation into the lipid bilayer membrane as discussed in a previous paper (Studer et al. 2009). Melittin-formed pores can be detected as intermediate current peaks. The lag time depends on the peptide concentration and the peak height can vary. The trace in Fig. 7b corresponds to previously observed data and a break after about $130 \mathrm{~min}$, due to a high number of pores, further indicates that a lipid bilayer membrane has been formed.

\subsubsection{Ion channel activity determination}

Integration of the membrane protein into lipid bilayer membranes formed in micropores is one of the most difficult steps (Zagnoni et al. 2007). It is well known that electrostatic repulsion, due to charged lipid head groups, prevents fusion of bilayers, e.g. of two liposomes. This unwanted repulsion can be minimized at high ion concentration, since the cations are shielding the negative charges. We used here a $3 \mathrm{M} \mathrm{KCl}$ solution to facilitate fusion of proteoliposomes with the preformed painted lipid 

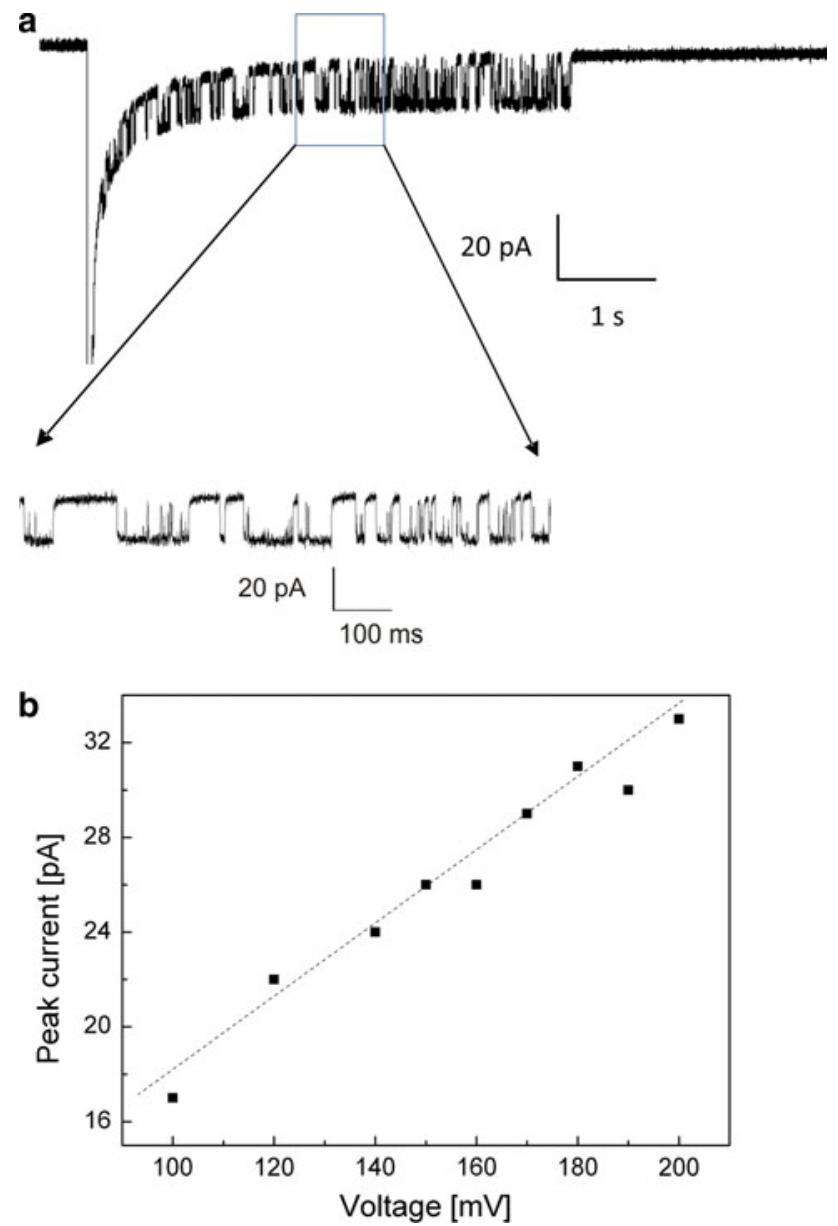

Fig. 8 Membrane protein activity. A lipid bilayer membrane was generated across the micropore by the painting method (DOPG:POPE $1: 3(\mathrm{v} / \mathrm{v}), 10 \mathrm{mg} / \mathrm{ml}$ in decane). The archaebacterial ion channel KvAP was inserted through fusion of proteoliposomes to the lipid bilayer from above. Single channel events could be detected after varying the potential across the membrane from +100 to $-100 \mathrm{mV}$ (a). Plotting the applied potential against the measured peak current shows a linear behavior (b), typical for channels with simple open or closed states

bilayer. Proteoliposomes added in a small volume $(<5 \mu \mathrm{l})$ of this high density solution sink by gravity to the surface. However, this procedure requires a horizontal setup. A previously presented alternative procedure, based on the nystatin-ergosterol system (Zagnoni et al. 2007), is orientation independent, however, shows other limitations, such as predefined lipid composition and low fusion rates. Furthermore, it is limited to pores larger than $6 \mu \mathrm{m}$ in diameter (Studer et al. 2011). In the future, more effective methods using suitable surface modifications can further improve this step and the transport of small volumes containing proteoliposomes to a micropore can also be controlled by the microfluidic system.

$\mathrm{KvAP}$ is an archaebacterial voltage-gated ion channel which opens upon changes in the applied potential.
Consequently, ions flow across bilayers and intermediate current steps of some pA-heights and milliseconds duration can be detected. The prerequisite for measuring such low currents are demanding. (1) The membrane sealing should be in the $\mathrm{G} \Omega$-range to reduce the noise below detection limit of single channels. Free-standing lipid bilayers are per se defect-free and exhibit a sealing (Fig. 6) sufficiently high for single ion channel measurements. (2) The electrical capacitance $(C)$ of the support should be low. Since $C$ is inversely related to the thickness of the support, silicon nitride membranes of $300 \mathrm{~nm}$ thickness have at least a ten times higher capacitance value than polymer foils of $12 \mu \mathrm{m}$ thickness. Therefore, a steep decrease within a few milliseconds of the discharging current after a potential change is observed (Fig. 8a) using the PEEK device. This is in sharp contrast to thin silicon nitride membranes, which have capacitance values of a few hundred $\mathrm{pF}$ (Han et al. 2007). Furthermore, the discharging current in these experiments are slow, making it impossible to detect currents in the pA-range within the short time period after the potential change when single channels open. In addition, the opening and closing of voltage-gated ion channels is stochastic. The blow-up in Fig. 8a shows that this particular KvAP ion channel can remain open for more than $100 \mathrm{~ms}$. The current height depends linearly on the applied voltage within the range from +100 to $+200 \mathrm{mV}$ (Fig. 8b) and the ion strength. The measured current peaks correspond to a single channel conductance of $170 \mathrm{pS}$ as reported (Ruta et al. 2003). This value for the reconstituted $\mathrm{KvAP}$ is also comparable to $260 \mathrm{pS}$ reported for a $\mathrm{Ca}^{2+}$-sensitive $\mathrm{K}^{+}$channel measured in $250 \mathrm{mM}$ buffer in oocytes (Perez et al. 1994). It can be concluded that single ion channels can be recorded using the here described PEEK device. Furthermore, the noise of about $4 \mathrm{pA}$ is sufficiently low to measure single ion channels but, it requires the measurement cell to be shielded from outside disturbances (Faraday cage).

\section{Conclusion}

We have demonstrated the fabrication of a versatile PEEKbased microfluidic device prepared by thermal bonding of PEEK foils comprising a micropore and a microfluidic channel structure, respectively. The mechanical stability of $12 \mu \mathrm{m}$ thin PEEK foils is sufficient for micropore generation by pulsed laser ablation and subsequent bonding to $300 \mu \mathrm{m}$ thick foils containing the microfluidic channel. The fabrication processes for such devices are relatively simple, and first results demonstrate the feasibility of the concept, i.e. these devices can be used as tools to measure activities of membrane proteins integrated in planar freestanding lipid bilayers. In principle, any membrane protein 
of interest can be integrated using available methods (Demarche et al. 2011). Optimization of the manufacturing processes will allow us to further exploit the potential of the presented concept.

Based on these findings, polymer-based bioanalytical systems with parallel detection of membrane proteins can be realized (Müller et al. 2011). Such devices are useful tools for monitoring the activity of membrane proteins relevant in research and drug discovery.

Acknowledgments The authors thank Armin Stumpp (FHNW) for support with pulsed laser ablation, Jens Gobrecht and Helmut Schift for discussing the concept and Mirco Altana and, Konrad Vogelsang (all from Laboratory for Micro- and Nanostructures, PSI) for help with hot embossing. The support of Patrick Schulz (MPIBP) in preparing and providing KvAP samples and KvAP-gene harboring vectors, Raimund Dutzler (University of Zurich) for kind help regarding liposome fusion, Valerie Panneels (BMR) for protein expression and purification and Sophie Demarche and David Langenegger (BMR) for experimental support and discussions is appreciated. This work was partially funded by the EU FP7 project ASMENA CP-FP 214666-2.

Open Access This article is distributed under the terms of the Creative Commons Attribution License which permits any use, distribution, and reproduction in any medium, provided the original author(s) and the source are credited.

\section{References}

Danelon C, Perez JB, Santschi C, Brugger C, Vogel H (2006) Cell membranes suspended across nanoaperture arrays. Langmuir 22:22-25. doi:10.1021/la052387v

Demarche S, Sugihara K, Zambelli T, Tiefenauer L, Vörös J (2011) Techniques for recording reconstituted ion channels. Analyst 136:1077-1089. doi:10.1039/c0an00828a

Estes DJ, Lopez SR, Fuller AO, Mayer M (2006) Triggering and visualization the aggregation and fusion of lipid membranes in microfluidic chambers. Biophys J 91:233-243. doi:10.1529/ biophysj.105.076398

Han X, Studer A, Sehr H, Geissbühler I, DiBerardino M, Winkler FK, Tiefenauer L (2007) Nanopore arrays for stable and functional free-standing lipid bilayers. Adv Mater 19:4466-4470. doi: 10.1002/adma.200700468

Hirano-Iwata A, Niwano M, Sugawara M (2008) The design of molecular sensing interfaces with lipid-bilayer assemblies. Trends Anal Chem 27(6):512-520. doi:10.1002/smll.201000997
Kawano R, Osaki T, Sasaki H, Takeuchi S (2010) A polymer-based nanopore-integrated microfluidic device for generating stable bilayer lipid membranes. Small 6(19):2100-2104. doi:10.1002/ smll.201000997

Kresak S, Hianik T, Naumann RLC (2009) Giga-seal solvent-free bilayer lipid membranes: from single nanopores to nanopore arrays. Soft Matter 5(20):4021-4032. doi:10.1039/b907661a

Malmstadt N, Nash MA, Purnell RF, Schmidt JJ (2006) Automated formation of lipid-bilayer membranes in a microfluidic device. Nano Lett 6(9):1961-1965. doi:10.1021/n10611034

Mayer M, Kriebel JK, Tosteson MT, Whitesides GM (2003) Microfabricated teflon membranes for low-noise recordings of ion channels in planar lipid bilayers. BiophysJ 85(4):2684-2695. doi:10.1016/S0006-3495(03)74691-8

Meier W, Graff A, Diederich A, Winterhalter M (2000) Stabilization of planar lipid membranes: a stratified layer approach. Phys Chem Chem Phys 2(20):4559-4562. doi:10.1039/b004073h

Müller E, Imhof I, Tiefenauer L, Schift H (2011) A method for producing a polymer-based microfluidics system for bioanalytics using biological membranes. Switzerland, Patent Appl. No. 11 163710.4 Patent

Nielsen CH (2009) Biomimetic membranes for sensor and separation applications. Anal Bioanal Chem 395(3):697-718. doi:10.1007/ s00216-009-2960-0

Perez G, Lagrutta A, Adelman JP, Toro L (1994) Reconstitution of expressed K-Ca channels from xenopus-oocytes to lipid bilayers. Biophys J 66(4):1022-1027

Ruta V, Jiang YX, Lee A, Chen JY, MacKinnon R (2003) Functional analysis of an archaebacterial voltage-dependent $\mathrm{K}^{+}$channel. Nature 422(6928): 180-185

Studer A, Han XJ, Winkler FK, Tiefenauer LX (2009) Formation of individual protein channels in lipid bilayers suspended in nanopores. Colloids Surf B Biointerfaces 73(2):325-331. doi: 10.1016/j.colsurfb.2009.06.006

Studer A, Demarche S, Langenegger D, Tiefenauer L (2011) Integration and recording of a reconstituted voltage-gated sodium channel in planar lipid bilayers. Biosens Bioelectron 26(5):1924-1928. doi:10.1016/j.bios.2010.06.008

Suzuki H, Takeuchi S (2008) Microtechnologies for membrane protein studies. Anal Bioanal Chem 391(8):2695-2702. doi: 10.1007/s00216-008-1916-0

Tiefenauer LX, Studer A (2008) Nano for bio: nanopore arrays for stable and functional lipid bilayer membranes (Mini Review). Biointerphases 3(2):FA74-FA79. doi:10.1116/1.2912932

Zagnoni M (2012) Miniaturized technologies for the development of artificial lipid bilayer systems. Lab Chip 12. doi:10.1039/c2lc20991h

Zagnoni M, Sandison ME, Marius P, Lee AG, Morgan H (2007) Controlled delivery of proteins into bilayer lipid membranes on chip. Lab Chip 7(9):1176-1183. doi:10.1039/b703818f 